\title{
O AGENTE de SAÚdE NA ATENC̣Ão PRIMÁRIA de SAÚdE DA TEORIA À REALIDADE
}

Maria da Glória Miotto Wright*

\begin{abstract}
RESUMO - A estratégia dos cuidados primários de saúde visa dar ao indivíduo, família e comunidade acesso universal a assistência de saúde essencial com baixo custo e com a participação da comunidade. O primeiro contato com os indivíduos e o sistema de saúde é oferecido pela agente de saúde comunitária. Sua função no sistema é fazer funcionar dinamicamente o sistema primário como o elo entre a comunidade e o sistema de cuidado primário de saúde. $\mathrm{O}$ estudo foi conduzido para saber o que a comunidade conhece sobre "agentes comunitários de saúde" e sobre o seu trabalho na comunidade e no Centro de Saúde. Depois de seis anos de experiência com o sistema de atuação primária de saúde, em Brasília, na Vila Paranoá, somente $21 \%(42)$ das famílias sabiam sobre o trabalho dos agentes comunitários de saúde. Os resultados deste estudo indicam a necessidade de repensar o papel, os métodos de trabalho e de treinamento dos agentes.
\end{abstract}

\begin{abstract}
Primary Health Care strategy aims to give individuals, families and the Community, universal access to essencial health assistance at low-cost and with community participation. In the inicial contacts between individuals and the health system, primary care is offered by the "Community Health Agent". His function in the primary Health system is to make the basic health services dynamic functioning as the "link" between the Community and the primary health care system. This study was conduct to learn what the Community Knows about the "Community Health Agent" and about their work in the Community and in the Health Center. After six years of experience with a primary Health Care System in Brasilia, in the Paranoá Vila, only $21 \%(42)$ of the families know about the Community Health Agents work. (The results of this study indicate a need to rethink the agent's role and methods of work and training.
\end{abstract}

\section{INTRODUC̣ÃO}

$\mathrm{Na}$ atualidade, existe uma grande desigualdade entre a of erta de serviços e o volume e a complexidade das necessidades de saúde.

Tradicionalmente, a comunidade tem expressado suas necessidades de saúde em termos dos problemas que devem ser atendidos com urgência: abastecimento de água, alimentos, atenção ao parto, acidentes e enf ermidades mais freqüentes, dentre as quais se encontram as transmissíveis. Desta forma, a maneira com que as comunidades percebem e atendem suas necessidades de saúde dif erem da forma em que as identifica e interpreta o sistema institucional (OPAS/OMS, 1977).
Dentro de um contexto de desenvolvimento integral, o conceito de cobertura de saúde, ultrapassa os limites tradicionais de uma simples proposição numérica, ela deve responder harmonicamente aos interesses nacionais, regionais e locais.

Diante dessa situação, foi reconhecida a necessidade de desenvolver uma nova estratégia, que corrigindo as limitações das ações desenvolvidas até recentemente para melhoria das condições de saúde da população, permita integrar todos os elementos necessários para conseguir um impacto real no estado de saúde da população (ROBAYO, 1976).

Esta nova estratégia é o que se chamou de "Atenção Primária", a qual representa uma abordagem prática para dar, aos indivíduos e às famílias da comuni-

* Docente Doutor, Adjunto III da Universidade de Brasília, Departamento de Enfermagem. 
dade, acesso universal aos cuidados essenciais de saúde, de forma aceitável, a um custo que esteja ao alcance e mediante a participação da comunidade (OMS/UNICEF, 1978).

\subsection{Agentes de saúde da comunidade - a concepção teórica}

A participação comunitária na fixação de políticas e em matéria de planejamento, execução e controle de programas de desenvolvimento é hoje uma prática amplamente aceita.

Dentro do contexto de Atenção Primária de Saúde, é de fundamental importância, o aproveitamento, integral de todos os recursos disponíveis, inclusive o potencial humano.

Ao nível inicial de contato entre os indivíduos e o sistema de serviços de saúde, os cuidados primários são of erecidos por Agentes de Saúde da Comunidade, trabalhando em equipe.

O objetivo fundamental da utilização do agente de Saúde é a dinamização do sistema da rede básica de serviços de saúde, funcionando como "elo" de ligação entre o sistema institucionalizado de saúde e a comunidade, tendo como base as atividades extramurais no sentido de procurar satisfazer as necessidades da população no próprio local onde vivem.

E vantajoso, que esses agentes de saúde, não só sejam naturais da comunidade em que vivem, como também, sejam escolhidos pela mesma. Recebendo treinamento elementar e a curto prazo, preparam-se para desempenhar as atividades correspondentes às necessidades expressas pela própria comunidade. À medida da necessidade, esse treinamento pode ser gradativamente ampliado. Além disso, devem receber um treinamento adequado, no que se refere à "Educação para Saúde", já que grande parte de seu tempo será dedicado à educação, bem como, devem ser capazes de reconhecer problemas e necessidades que estejam fora de sua área de ação e levá-los à outros níveis para solucioná-los. (ROBAYO, 1976).

Para que os agentes de saúde desempenhem um papel cada vez mais importante na oferta de cuidados primários de saúde, eles devem ser retreinados após seu treinamento inicial (OMS/UNICEF, 1978).

As características essenciais do Agente de Saúde são (coletanea contact, 1979):

Quem é o Agente de Saúde?

É um homem ou uma mulher, que na medida do possível sabe ler e escrever e é selecionado pelas autoridades da comunidade, ou com a sua aprovação, para lidar com problemas de saúde individuais e comunitários;

A quem está subordinado?

0 agente de saúde será subordinado tanto perante as autoridades locais como perante um supervisor oficial. Poderá receber seu pagamento diretamente da comunidade ou da Instituição de Saúde, à qual estará filiado. Seu trabalho poderá ser de tempo integral ou parcial.

Que tipo de treinamento deve receber?

0 agente de saúde receberá um treinamento inicial de seis a oito semanas de duração. Depois deverá receber um treinamento suplementar periódico, de duas ou três semanas de duração. Seu treinamento será prático e ministrado na própria comunidade.

Quais serão suas tarefas?

0 trabalho do agente de saúde, cobrirá tanto a área de assistência à saúde, como a de desenvolvimento comunitário. As ações que os agentes de saúde poderiam desenvolver são:

- Cuidar da saúde dos habitantes e zelar pela higiene na comunidade;

- Dar assistência e orientações, de acordo com as instruções do supervisor, a qualquer habitante que o consulte;

- Encaminhar os pacientes ao Centro de Saúde ou ao hospital mais próximo;

- Deve visitar todas as moradias e dar aos seus moradores orientações como prevenir doenças e ensinar bons hábitos de higiene;

- Enviar relatórios periódicos à autoridade local sobre a saúde dos habitantes e as condições de higiene da comunidade;

- Solicitar das autoridades e do povo a ajuda e o apoio de que necessita em seu trabalho;

- Promover atividades de desenvolvimento comunitário e desempenhar papel ativo nelas;

- Manter-se sempre em contato com o supervisor imediato.

\subsection{Os programas de extensão de cobertura de serviços de saúde no Brasil - antecedentes e perspectivas}

Somente a partir da segunda metade da década de 70, foram implantados no Brasil os Programas de Extensão de Cobertura, originários do Movimento da Medicina Comunitária: "destinam-se à prestação de serviços sanitários, aí incluída a atenção médica individual, às populações carentes de áreas rurais marginalizadas dos centros urbanos, valendo-se para tanto de modelos particulares de organização dos serviços, da simplificação tecnológica e do recurso à participação comunitária. (DONNANGELO, 1976). Nesse sentido, o movimento da Medicina Comunitária, além de ter introduzido uma concepção de como se articulam medicina e sociedade, influencia na reorganização da prática médica e se apresenta como uma alternativa para a crise do setor de saúde, desencadeada pelo modelo econômico vigente no país. 
Estas reformulações, ao nível da prestação dos serviços de saúde, implicariam numa nova maneira de se conceber o recurso humano que viesse a atuar nos serviços de saúde, com base na proposta de simplificação da Medicina.

Esse tipo de programa tem sido experimentado em diversas Unidades Federadas. A Fundação de Serviços Especiais de Saúde Pública (FSESP), do Ministério da Saúde, além de trabalhar com parteiras leigas orientadas, já vem há muito tempo, operando suas unidades com a utilização intensiva de pessoal de nível auxiliar de enfermagem, em unidades onde não há médico.

Dentre os programas de maior abrangência destacase o Programa de Interiorização de Ações de Saúde e Saneamento do Nordeste-PIASS, certamente o esforço mais amplo que já se fez no brasil em matéria de interiorização de ações de saúde com utilização de pessoal auxiliar. Outros programas que surgiram também, foram os Programas de Desenvolvimento Regionais Integrados, com o objetivo de extensão de cobertura a baixo custo, com ações de saúde simplificadas dentro de uma hierarquização de serviços (BRASIL, 1979).

A formação e absorção de Recursos Humanos para tais programas, surge em 1975 com o Programa de Preparação Estratégica de Pessoal para Saúde (PPREPS), criado através dos Ministério da Saúde, Educação e Cultura e da Organização Pan-Americana de Saúde com o propósito de "adequar progressivamente a formação desses recursos humanos para a saúde com cobertura máxima possível e integral, regionalizado e de assistência progressiva, de acordo com as necessidades das populações respectivas e as possibilidades das diversas realidades que o país apresenta" (BRASIL, MS/MEC/OPAS, 1976).

Nos meados de 1980, surge o PREV-SAÚDE (Brasil, MS, MPAS, 1981) Programa de Serviços Básicos de Saúde, proposto para ser o programa axial e nuclear das ações de saúde do governo, de caráter interministerial, em função do qual deverão orientar-se os demais programas e atividades do setor saúde. Entretanto, as proposições contidas nas várias versões do PREVSAÚDE não chegaram a se concretizar, embora em muitos estados se evidenciou a implantação da extensão de cobertura de saúde, através da Atenção Primária, como foi o caso, em particular, do Distrito Federal.

\subsection{A implantação da rede de serviços básicos de saúde no Distrito Federal}

Quando, em 1980, o então Secretário da Saúde, assumiu a secretaria de Saúde do governo do Distrito Federal, a situação saúde e de assistência à saúde estava um verdadeiro caos. Partindo, da análise do Sistema de Saúde existente até então no Distrito Federal e tomando conhecimento dessas deficiências passou a meta governamental a extensão de cobertura de serviços básicos de saúde através de uma hierarquização com níveis de complexidade crescentes da assistência, o que compreendeu a regionalização da assistência (Brasília, Secretaria da Saúde, 1980).

Cada cidade satélite e o plano piloto, recebeu tantos postos e centros de saúde (Nível Primário) em conformidade com o total de população existente em cada localidade e mais um hospital regional (Nível Secundário). $\mathrm{O}$ sistema de referência final compreendeu 0 Hospital de Base de Brasília, cujo atendimento é a Nível Terciário, com todas as subespecialidades.

Nessa ocasião foram implantados 40 Centros e Postos de Saúde e os Hospitais Regionais das cidades satélites e Plano Piloto, onde estes ainda não existiam.

Dentro desta nova filosofia, foram recrutados para trabalhar nos postos e centros de saúde os "Agentes de Saúde da Comunidade". O número desses agentes em cada unidade de atenção primária de saúde estava em relação direta com o número total da população que deveria receber este tipo de serviço.

Após o recrutamento (o qual se constituia numa prova de seleção), 555 candidatos foram classificados para a função de agentes de Saúde Pública. Eles receberam um treinamento de 8 semanas fora do local onde iriam atuar e foram distribuídos entre os postos e centros de saúde, obedecendo de certa forma, seu local de origem. Coube ao pessoal de Enfermagem e de Serviço Social a maior parte do trabalho de formação do agente de Saúde. As disciplinas e a carga horária que compunham esse treinamento foram:

$$
\begin{array}{ll}
\text { - Serviço social } & 40 \text { horas } \\
\text { - Nutrição } & 14 \text { horas } \\
\text { - Vigilância } & 12 \text { horas } \\
\text { - Educação para Saúde } & 10 \text { horas } \\
\text { - Enfermagem } & 84 \text { horas } \\
\text { - Estágio } & \underline{80 \text { horas }} \\
\cline { 2 - 2 } \text { Total } & 240 \text { horas }
\end{array}
$$

(entre teoria e prática)

0 recrutamento foi feito para dois níveis de agente: Agente de Saúde Pública nível $A$ (possuir escolaridade de $1^{\circ}$ grau) e o nível $B$ (possuir escolaridade de 2 ? grau). Isto levou a um nível desigual de salário para a função Agente de Saúde Pública (Brasília, Secretaria da Saúde, Fundação Hospitalar do Distrito Federal - CISB, 1980).

\section{JUSTIFICATIVA}

Com a implantação da rede de serviços básicos de saúde e a adoção da assistência primária de saúde, como uma metodologia alternativa, o agente de saúde da comunidade, surge para dinamizar esse novo sistema em expansão em algumas Unidades Federativas do País.

Durante todos esses anos de implantação e implementação desses programas, surgiram vários questionamentos no que diz respeito à prática desses agentes, tanto no que se refere à necessidade de se ampliar o rol de suas funções originais, como nas supostas atri- 
buições de que o seu desempenho é o responsável direto pela eficiência ou não dos serviços de saúde desenvolvidas nos postos centros de saúde.

Este tipo de questionamento, está até certo ponto, ligado ao processo de capacitação dos agentes de saúde, responsáveis pelo atendimento primário, no que refere à defazagem dos conteúdos e método pedagógico utilizado, período e local de treinamento, o rol de funções e o próprio perfil dos agentes de saúde.

Levando-se em conta, que o órgão formador do Agente de Saúde da comunidade é, quase sempre, o seu utilizador a proposta de desenvolver um estudo que mostre a visão da comunidade em relação a esse novo elemento dentro da equipe de saúde, bem como a sua inserção no próprio sistema de Atenção Primária de Saúde, poderá contribuir para a formulação das bases teóricas e práticas dos programas de retreinamento desses agentes de saúde da comunidade.

Além disso, este estudo é justificado também, porque temos acompanhado, desde a implantação, o desenvolvimento das atividades dos agentes de saúde em uma comunidade periurbana do Distrito Federal.

\section{METODOLOGIA}

\subsection{Descrição da área}

A área escolhida para desenvolver o estudo em relação aos Agentes de Saúde, foi a Vila do Paranoá. Esta se localiza a $26 \mathrm{~km}$ do Plano Piloto, próximo à barragem do Lago Paranoá, existindo desde 1957, com a construção do acampamento para execução das obras da barragem.

A Comunidade do Paranoá conta atualmente com mais de 20.000 habitantes e se divide em dois núcleos principais, sendo o primeiro aquele chamado "antigo acampamento da construção da barragem" e o segundo a "Invasão do Paranoá", sendo que esta se subdivide em "Vila Piaui" e "Vila Paranoá". A Vila Paranoá se originou de invasões, os residentes montaram seus próprios barracos e moram neles, estando livres de pagamento de aluguel ou tributos. Há energia elétrica, um posto da Legião Brasileira de Assistência (LBA), uma escola de primeiro grau, um centro de saúde, um centro da Secretaria do Serviço Social, um curso supletivo à noite e um posto policial. Além disso, tem acesso a hospitais no Plano Piloto e distâncias de transportes inferiores às das “cidades satélites"'. Não há, entretanto, infra-estrutura apropriada no tocante a água e esgoto.

\subsection{Amostra}

A mostra foi constituída de 260 domicílios selecionados aleatoriamente DE UM TOTAL DE 2.600 domicílios cadastrados na TERRACAP.

\subsection{Instrumento}

Para a coleta de dados dessa pesquisa, foi utilizado um formulário contendo perguntas abertas e fechadas e foi aplicado através de entrevista.

\section{RESULTADOS E ANÁLISE}

Dos 260 domicílios selecionados, conseguimos fazer entrevistas com 198 famílias. Os 62 domicílios restantes que não foram entrevistados correspondiam à Igrejas, bares, ou residências fechadas no momento das entrevistas. Apenas 196 famílias responderam às questões sobre os agentes de saúde.

Os resultados mostraram que do total de 196 famílias, apenas $37,7 \%$ (74) disseram que conhecem o agente e $62,3 \%$ (122) não os conhecem. Ao serem questionadas sobre se sabem o que os agentes fazem, apenas $21,4 \%$ (42) responderam afirmativamente e 78,6\% (154) desconhecem o que eles fazem.

A Tabela l é a combinação das respostas afirmativas e negativas do conhecimento da comunidade sobre o Agente de Saúde do Centro de Saúde n. 15 e o que ele costuma fazer. Apenas 21,4\% (42) das famílias conhecem e sabem o que os Agentes de Saúde fazem, $16,3 \%$ das familias disseram que os conhecem, mas não sabem o que eles fazem e um total de $62,3 \%$ (122) não os conhecem e também não sabem o que eles fazem nem no centro de saúde $n^{0}$. 15 bem como na própria comunidade.

TABELA 1 - Conhecimento da comunidade sobre o agente de saúde e o que ele faz.

\begin{tabular}{|c|c|c|c|c|c|c|c|}
\hline \multirow{2}{*}{$\begin{array}{cc}\text { Pergunta } & \text { Pergunta } \\
& \text { Respostas } \\
\end{array}$} & & \multicolumn{6}{|c|}{ A Comunidade Conhece o Agente de Saúde? } \\
\hline & & \multicolumn{2}{|c|}{ SIM } & \multicolumn{2}{|c|}{ NÃO } & \multicolumn{2}{|c|}{ TOTAL } \\
\hline $\begin{array}{l}\text { A comunidade sabe } \\
\text { o que faz } \\
\text { o Agente de Saúde? }\end{array}$ & $\begin{array}{c}\text { SIM } \\
\text { NÃO } \\
\text { TOTAL }\end{array}$ & $\begin{array}{l}42 \\
32 \\
74\end{array}$ & $\begin{array}{l}21,4 \% \\
16,3 \% \\
37,7 \%\end{array}$ & $\begin{array}{r}0 \\
122 \\
122\end{array}$ & $\begin{array}{c}0,0 \\
62,3 \% \\
62,3 \%\end{array}$ & $\begin{array}{r}42 \\
154 \\
196\end{array}$ & $\begin{array}{r}21,4 \% \\
78,6 \% \\
100,0 \%\end{array}$ \\
\hline
\end{tabular}


Na Tabela 2 encontramos o relato de algumas das atividades mencionadas por $21,4 \%$ (42) das famílias sobre o que os agentes de saúde fazem tanto no Centro de Saúde n? 15 - Paranoá como na Comunidade. Na Comunidade do Paranoá, (atividade externa), o agente de saúde faz, basicamente dois tipos de atividade: a visita domiciliária com suas diversas finalidades, $\mathrm{e}$ o outro tipo de atividade identificada pelas famílias foi a participação deles em levantamentos e pesquisa.

No Centro de Saúde, local destinado para a realização de serviços internos, a gama de atividades mencionadas pelas famílias, incluem ações desenvolvidas que vão desde a sala de farmácia, sala de curativos, sala de vacinação, triagem, até exame físico, assistência à gestante e criança, encaminhamento de pacientes aos médicos e hospital.

Para iniciar alguma análise destes resultados, é importante salientar, que os estudos que baseiam a análise das práticas e a formação dos Agentes de Saúde na Atenção Primária de Saúde, à nível de Brasil, são raros. Os estudos realizados nesta área, estão voltados principalmente para os recursos humanos de nivel superior (Brasil, MEC/DAU, 1976; MACEDO, 1982; DONNANGELO, 1976).

$\mathrm{Na}$ concepção filosófica da Atenção Primária de Saúde, a participação da comunidade é vista como uma chave fundamental para a implantação do programa. Essa ideologia gerou a expectativa de que as pessoas da comunidade são um grande potencial de recursos não explorado e, se mobilizado, pode contribuir para o escasso conjunto dos recursos de saúde para reduzir o custo do atendimento, fornecendo mão-de-obra suplementar (RIKFIN, 1984).

Assim, a idéia de introduzir na equipe de saúde o elemento "Agente de Saúde" leva os planejadores a vê-los como o representante-chave dos pontos de vista da comunidade, além de serem considerados também como "agentes de mudança".

Após seis anos de implantação da Atenção Primária de Saúde no Distrito Federal, encontramos um quadro, que apesar de representar apenas uma comunidade, onde a realidade é bem diferente da proposta teórica preconizada sobre a participação dos Agentes de Saúde no sistema de extensão de cobertura de serviços de saúde à população.

Segundo os dados apresentados nesta pesquisa, apenas $21,4 \%$ (42)das famílias entrevistadas, tanto conhecem como sabem de alguma atividade que os agentes de saúde realizam. RIF KIN (1984) comenta que, nos programas de "Saúde Comunitária e Desenvolvimento (SCD)", iniciados em alguns países na década de 70, "muitos dos membros da Comunidade não tinham co-

TABELA 2 - Visão da comunidade sobre o tipo de atividade desenvolvida pelo agente de saude.

\section{LOCAL}

1 - Na Comunidade (serviço externo)

2 - No Centro de Saúde (serviço interno)

\section{TIPO DE ATIVIDADE}

a) - Realização de Visitas Domiciliares para:

- Acompanhamento de gestante e recém-nascidos;

- Avisar sobre vacinas atrasadas;

- Entregar cartão consulta;

- Avisar sobre o dia da consulta;

- Atendimento domiciliar de emergência;

- Dar orientação sobre saúde e higiene em geral.

b) - Participação de levantamentos e pesquisas.

- Atendimento na farmácia;

- Fazem exame físico;

- Aplicam Injeção;

- Distribuição de fichas aos pacientes;

- Atuam na vacinação;

- Fazem curativos;

- Encaminhamento dos pacientes aos médicos;

- Marcam consultas;

- Acompanhamento do paciente ao hospital;

- Atendem crianças e gestantes;

- Fazem atividades de enfermagem;

- Consultam os pacientes; 
nhecimento do agente ou só viam neles um distribuidor de remédios", atividade esta, também mencionada nos achados de nosso estudo.

0 que se percebe é que existe tanto por parte dos agentes, como da comunidade, dificuldade em entender o ponto de vista da filosofia imperante no modelo de Assistência Primária. Essa dificuldade, não é de responsabilidade deles e, sim, da parte dos planejadores, que gostam de atropelar as fases de implantação de um projeto na comunidade, escamoteando a face de autoritarismo e política existente por parte do Estado. 0 processo não se diz democrático simplesmente porque revela participação, pois, como vimos, a participação pode ser o primeiro momento da manipulação política. Assim, tanto quanto o grau de participação, importa conhecer o produto, o resultado, vale dizer, seu conteúdo; em outras palavras, os objetivos políticos aos quais a participação serve, ou está servindo.

De acordo com os resultados da Tabela 4, onde mostra a visão da comunidade sobre o tipo de atividade desenvolvida pelo agente de saúde à nível de comunidade e no Centro de Saúde, fica claro que os resultados atuais estão longe do arcabouço teórico preconizado para este novo elemento da equipe de saúde. À nível externo, é enfatizado teoricamente que a ênfase de seu serviço deve ser a "educação para a saúde", e nossos resultados indicam que as visitas domiciliares são usadas em sua maior parte para: avisar que as vacinas estão atrasadas, entregar cartão de consulta, avisar sobre o dia da consulta ou então, atendimento domiciliar de emergência. Aquilo que poderíamos considerar como educação para saúde, é conhecido pela comunidade de acompanhamento de gestante e recémnascido e orientações sobre ' saúde em geral e higiene', que é realizado em proporção menor às outras atividades.

A nível de Centro de Saúde, as tarefas realizadas pelos agentes as quais a comunidade identificou, atuaram contra o pleno desempenho de seu papel. Para a comunidade, o agente de saúde é identificado como um dos elementos da equipe de enfermagem, fazendo, às vezes, o papel do atendente, auxiliar, técnico e até mesmo do enfermeiro. Indicando com isto, que a maior parte do tempo desses agentes é gasto em atividade curativa GABARRA (1980) e SILVA (1983) encontraram resultados semelhantes no que se refere ao tempo gasto com atividade externa e interna, além das ações educativas serem reduzidas e realizadas de forma incidental. 0 que vem, também, demonstrar uma distorção na adequação da prática com o treinamento recebido pelos agentes de saúde.

\section{CONCLUSÃO}

Assim, após 6 anos de atuação dos agentes de saúde na Atenção Primária de Saúde no Distrito Federal, encontramos o agente, sendo identificado pela comu- nidade como um prestador de serviços, que depende do profissional de saúde para orientação e definição de seu papel, tanto à nível de comunidade como à nível de centro de saúde. Por outro lado, a comunidade tem a tendência de ver o agente, não como um fator de mudança, mas considerá-lo simplesmente como um membro da comunidade que está ligado à Associação dos Moradores da Comunidade. Deste modo, o despertar da consciência para uma mudança, foi estorvado pela expectativa de atividades curativas.

Alguns pontos podemos levantar, sobre o que poderia ter contribuído para esse conhecimento insuficiente da comunidade sobre o agente de saúde:

- Os planejadores, usando basicamente a abordagem médica, porém com uma retórica de participação comunitária, deram um papel dominante aos profissionais, principalmente aos profissionais da Medicina; - Os médicos dos centros de saúde, apesar de serem peças-chave para a saúde comunitária, estão interessados em limitar-se a prestar serviços;

- Na tentativa de alcançar a participação comunitária o programa de Atenção Primária de Saúde introduziu os "Agentes de Saúde", que além de ser o elo de ligação do serviço com a comunidade deveria desenvolver a participação da comunidade. Entretanto, seus papéis nunca foram claramente definidos, nem para a equipe de saúde, nem para a comunidade;

- O sistema utilizado para o recrutamento dos Agentes de Saúde não contou com a participação da comunidade, e as pessoas que se candidataram, em sua maioria, não tinham atuação ativa na comunidade;

- O papel e o treinamento do agente de saúde, também reflete a preocupação com os serviços; pois concebido e executado por técnicos fora das comunidades de origem dos agentes, esse esquema não levou em consideração as necessidades e pontos de vista da comunidade e, como resultado, a comunidade pouco os conhece, além de estarem desempenhando atividades que não são de suas funções;

- Os planejadores procuravam enfrentar a necessidade de melhorar a atenção de saúde da população, sem pesquisar o que a população queria;

- Não houve uma fase de divulgação adequada para a comunidade do papel do Centro de Saúde, da Atenção Primária de Saúde, assim, quando este entrou em ação, muitos membros da comunidade não tinham o conhecimento desse novo elemento da equipe de saúde, além de muitos agentes não entenderem sua função como "agentes de mudança", eles se apoiaram na prestação de serviços;

- Inexistência de um sistema ef etivo e contínuo de supervisão direta das atividades dos agentes de saúde, por parte dos supervisores imediatos, o que gera a não sistematização do registro diário das atividades desenvolvidas, o que permitiria que todos os elementos da equipe de saúde saibam o que eles fazem e a evolução das condutas adotadas; 
- Inexistência de um sistema de retreinamento dos agentes de saúde, levando-se em conta a realidade de inserção desse elemento na equipe de saúde e as necessidades da população onde ele desenvolve seu trabalho;

- Inexistência de um sistema contínuo de avaliação do programa de Atenção Primária de Saúde no Distrito Federal, permitindo medir qualitativamente e quantitativamente seu ef eito na melhoria das condições de saúde da população.

Os resultados desse estudo em relação ao trabalho do agente de saúde na comunidade do Paranoá, ressaltam que a continuidade deles como uma peça importante no desenvolvimento da Atenção Primária de Saúde deve ser revista e repensado o seu papel, métodos de trabalho e treinamento, além de tentar buscar com eles e a Comunidade as soluções para os aspectos limitantes identificados.

\section{REFERÊNCIAS BIBLIOGRÁFICAS}

1. COLETÂNEA CONTACT. O auxiliar de Saúde, em: mudanças de papéis na equipe de saúde. São Paulo, Edições Paulinas. 1979.

2. DONNANGELO, M.C.F. Educação Sanitária e políticas de saú(le. São Paulo (mimeogr.) 1976.

3. (1976) - Saúde e Sociedade,São Paulo, Duas Cidades.

4. GABARRA, M.O. Estudo do tempo, de acordo com o tipo de atividade, utilizado pelas visitadoras de um centro de saúde. Revista Gaúcha de Enfermagem 2 (2,3,4): 91-104, 1980.

5. BRASÍlIA. Secretaria de Saúde. Plano de Assistência à Saúde no Distrito Federal e Atenção Primária em Saúde do Dis- trito Federal. Brasília, 2v. 1980.

6. BRASÍLIA. Secretaria da Saúde. FUNDAÇÃO HOSPITALAR DO DISTRITO FEDERAL. Centro Interescolar de Saúde de Brasília (CISB). Programa de Treinamento para Agente de Saúde Pública, Brasília, 1980.

7. MACEDO, C.G. - Recursos humanos para a saúde, Rio de Janeiro, Fundação Getúlio Vargas, Escola Brasileira de Administração Pública, PROASA. 1982.

8. BRASIL. Ministério da Saúde; Ministério da Educação e Cultura; Organizaçāo Panamericana da Saúde, Programa de Preparação estratégica de pessoal de saúde, Brasília. 1976.

9. BRASIL. Ministério da Saúde. Ministério da Previdência e Assistência Social. Programa Nacional de Serviços Básicos de Saúde (PREV-SAÚDE), Brasília. 1981.

10. BRASIL. Ministério da Educação e Cultura. Departamento de Assuntos Universitários. Estudo sobre a formação e utilização dos recursos humanos na área de saúde, Brasília. 1976.

11. ORGANIZACIÓN PANAMERICANA DE LA SALUD \& ORGANIZACIÓN MUNDIAL DE LA SALUD. Extensión de cobertura de los serviços de salud con las estrategias de atención primária y participación de la consunidade. IV Reunião Especial de Ministros de Salud de las Américas Washington, 2()-27 de sept. 1977.

12. ORGANIZAÇÃO MUNDIAL DE SAÚDE: FUNDO DAS NAÇŌES UNIDAS PARA A INFÂNCIA. Cuidados Primários de Saúde, Conferência Internacional sobre cuidados Primários de Saúde, Alma ATA, URSS, 6-12 de 1978.

13. BRASIL. Ministério da Saúde. Programa de Interiorização das Ações de Saúde e Saneamento - PIASS. Diretrizes e Estratégias para 1979.

14. RIKFIN, S.B. Participação comunitária na teoria e na realidade. 1984 .

15). R()BAY(), J.C. Situaçoes de cobertura, níveis de atenção primária. In: SEMINÁRIO S()BRE N()VAS I)IMENS()ES' NO PAPEL DA ENFERMAGEM NA PRESTAÇÃ() I)E ATENÇÄ() PRIMÁRIA. San José - Costa Rica. 1976.

16. SILVA, J. A. Assistência Primária de Sauide. - () Agente de Saúde (lo) Vale da Ribeira, São Paulo, I)iss. mest. 198:3. 